\title{
ON THE SPECTRUM OF WEAKLY ALMOST PERIODIC SOLUTIONS OF CERTAIN ABSTRACT DIFFERENTIAL EQUATIONS
}

\author{
ARIBINDI SATYANARAYAN RAO \\ Department of Mathematics \\ Sir Ceorge Williams Campus \\ Concordia University \\ Montreal, Quebec, Canada \\ and

\section{L.S. DUBE} \\ Department of Mathematics \\ Vanier College \\ 821 Ste-Mis Croix Blvd. \\ St. - Laurent \\ Quebec, H4L 3×9, Canada \\ (Received October 15, 1984)
}

\begin{abstract}
In a sequentially weakly complete Banach space, if the dual operator of a linear operator A satisfies certain conditions, then the spectrum of any weakly almost periodic solution of the differential equation $u^{\prime}=A u+f$ is identical with the spectrum of $f$ except at the origin, where $f$ is a weakly almost periodic function.

KEY WORDS AND PHRASES. Strongly (weakly) almost periodic function, sequentially weakly complete Banach space, densely defined linear operator, dual operator, Hilbert space, nonnegative self-adjoint operator.

1980 MATHEMATICS SUBJECT CLASSIFICATITON CODE. 344:5, 34G0\%; $43 A 60$
\end{abstract}

1. INTRODUCTION.

Suppose $X$ is a Banach space and $X^{*}$ is the dual space of $X$. Let $J$ be the interval $-\infty<t<\infty$. A continuous function $f: J \rightarrow X$ is said to be strongly almost periodic if, given $\varepsilon>0$, there is a positive real number $2=\ell(\varepsilon)$ such that any interval of the real line of length $\ell$ contains at least one point $\tau$ for which

$$
\sup _{t \varepsilon J}|| f(t+\tau)-f(t) \| \leq \varepsilon .
$$

We say that a function $f: J \rightarrow X$ is weakly almost periodic if the scalarvalued function $\left\langle x^{\star}, f(t)\right\rangle=x^{\star} f(t)$ is almost periodic for each $x^{\star} \varepsilon X^{\star}$.

It is known that, if $X$ is sequentially weakly complete, $f: J \rightarrow X$ is weakly almost periodic, and $\lambda$ is a real number, then the weak limit 


$$
m\left(e^{-i \lambda t} f(t)\right)=\underset{T \rightarrow \infty}{w-1} i m \frac{1}{T} \int_{0}^{T} e^{-i \lambda t} f(t) d t
$$

exists in $X$ and is different from the null element $\theta$ of $X$ for at most a countable set $\left\{\lambda_{n}\right\}_{n=1}^{\infty}$, called the spectrum of $f(t)$ (see Theorem 6, p. 43, Amerio-Prouse [1]). We denote by $\sigma(f(t))$ the spectrum of $f(t)$.

2. RESULTS

Our first result is as follows (see Theorem 9, p. 79, Amerio-Prouse [1] for the spectrumi of an $s^{1}$-almost periodic function).

THEOREM 1. Suppose $X$ is a sequentially weakly complete Banach space, $A$ is a densely defined linear operator with domain $D(A)$ and range $R(A)$ in $X$, and the dual operator $A^{\star}$ is densely defined in $X^{\star}$, with $R\left(i \lambda-A^{*}\right)$ being dense in $X^{\star}$ for all real $\lambda \neq 0$. Further, suppose $f: J \rightarrow X$ is a weakly almost periodic (or an $S^{1}$-almost periodic continuous) function. If a differentiable function $u: J \rightarrow D(A)$ is a weakly almost periodic solution of the aifferential equation

$$
u^{\prime}(t)=A u(t)+f(t)
$$

on $\mathrm{J}$, with $u^{\prime}$ being weakly continuous on $J$, then $\sigma(u(t)) \backslash\{0\}=\sigma(f(t)) \backslash\{0\}$.

PROOF OF THEOREM 1. First we note that $u$ is bounded on $J$, since $u$ is weakly almost periodic. Hence, for $X^{\star} \in X^{\star}$, we have

$$
\begin{aligned}
\frac{1}{T} \int_{0}^{T} e^{-i \lambda t} x^{\star} u^{\prime}(t) d t & =x^{\star} \frac{1}{T}\left\{\left[e^{-i \lambda t} u(t)\right] \int_{0}^{T}+\frac{i \lambda}{T} \int_{0}^{T} e^{-i \lambda t} u(t) d t\right\} \\
& \rightarrow i \lambda x^{\star} m\left(e^{-i \lambda t} u(t)\right) \text { as } T \rightarrow \infty .
\end{aligned}
$$

So, for $x^{\star} \in D\left(A^{\star}\right)$, it follows from (1.3) that

$$
\begin{aligned}
\lim _{T \rightarrow \infty} \frac{1}{T} \int_{0}^{T} e^{-\lambda t} x^{\star} A u(t) d t & =\lim _{T \rightarrow \infty} \frac{1}{T} \int_{0}^{T} e^{-i \lambda t}\left(A^{\star} x^{\star}\right) u(t) d t \\
& =\lim _{T \rightarrow \infty}\left(A^{\star} x^{\star}\right)\left[\frac{1}{T} \int_{0}^{T} e^{-i \lambda t} u(t) d t\right] \\
& =\left(A^{\star} x^{\star}\right) m\left(e^{-i \lambda t} u(t)\right) \\
& =i \lambda x^{\star} m\left(e^{-i \lambda t} u(t)\right)-x^{\star} m\left(e^{-i \lambda t} f(t)\right) .
\end{aligned}
$$

Consequently, we have

$$
x^{\star} m\left(e^{-i \lambda t} f(t)\right)=\left(i \lambda x^{\star}-A^{\star} x^{\star}\right) m\left(e^{-i \lambda t} u(t)\right) .
$$

Now suppose that $\lambda \varepsilon \sigma(f(t)) \backslash\{0\}$. Then, since $D\left(A^{\star}\right)$ is dense in $X^{\star}$, there exists $x_{1}^{\star} \in D\left(A^{\star}\right)$ such that

$$
0 \neq x_{1}^{*} m\left(e^{-\lambda t} f(t)\right)=\left(-\lambda x_{1}^{*}-A^{*} x_{1}^{*}\right) m\left(e^{-\lambda t} u(t)\right) \text {. }
$$


Therefore $m\left(e^{-i \lambda t} u(t)\right)=0$ and so $\lambda \varepsilon \sigma(u(t)) \backslash\{0\}$.

Thus we have

$$
\sigma(f(t)) \backslash\{0\}-\sigma(u(t)) \backslash\{0\} .
$$

Now assume that $\lambda \in \sigma\left(u(t) \backslash\{0\}\right.$. Then, since $R\left(i \lambda-A^{\star}\right)$ is dense in $x^{\star}$, there exists $x_{2}^{*} \in D\left(A^{*}\right)$ such that

$$
0=\left(-i \lambda x_{2}^{*}-A^{*} x_{2}^{*}\right) m\left(e^{-i \lambda t} u(t)\right)=x_{2}^{*} m\left(e^{-i \lambda t} f(t)\right) .
$$

Therefore $m\left(e^{-i \lambda t} f(t)\right) \neq 0$ and so $\lambda \varepsilon \sigma(f(t)) \backslash\{0\}$.

Consequently, we have

$$
\sigma(u(t)) \backslash\{0\} \quad \sigma(f(t)) \backslash\{0\} .
$$

It follows from (2.5) and (2.7) that $\sigma(u(t)) \backslash\{0\}=c(f(t)) \backslash\{0\}$, which completes the proof of the theorem.

REMARK 1. The conclusion of Theorem 1 remains valid if $D\left(A^{*}\right)$ is total and $R\left(i \lambda-A^{\star}\right)$ is total for all real $\lambda \neq 0$, instead of dense in $X^{\star}$.

We indicate the proof of the following result.

THEOREM 2. In a sequentially weakly complete Banach space $X$, suppose $A$ is a densely defined linear operator, the dual operator $A^{\star}$ is densely defined in $X^{\star}$, with $R\left(\lambda^{2}+A^{*}\right)$ being dense in $X^{\star}$ for all real $\lambda \neq 0$, and $f: J \rightarrow X$ is a weakly almost periodic (or an $S^{1}$-almost periodic continuous) function. If a twice differentiable function $u: J \rightarrow D(A)$ is a weakly almost periodic solution of the differential equation

$$
u^{\prime \prime}(t)=A u(t)+f(t)
$$

on $J$, with $u^{\prime \prime}$ being weakly continuous and $u^{\prime}$ bounded on $J$, then

$$
\sigma(u(t)) \backslash\{0\}=\sigma(f(t)) \backslash\{0\} \text {. }
$$

PROOF. For $x^{\star} \in D\left(A^{\star}\right)$, we have

$$
\begin{aligned}
& \frac{1}{T} \int_{0}^{T} e^{-i \lambda t} x^{\star} u^{\prime \prime}(t) d t=x^{\star}\left\{\frac{1}{T}\left[e^{-i \lambda t} u^{\prime}(t)\right]_{0}^{T}+\frac{i \lambda}{T} \int_{0}^{T} e^{-i \lambda t} u^{\prime}(t) d t\right\} \\
&=x^{\star}\left\{\frac{1}{T}\left[e^{-i \lambda t} u^{\prime}(t)\right]_{0}^{T}+\frac{i \lambda}{T}\left[e^{-i \lambda t} u(t)\right]_{0}^{T}-\frac{\lambda^{2}}{T} \int_{0}^{T} e^{-i \lambda t} u(t) d t\right\} \\
& \rightarrow-\lambda^{2} x^{\star} m\left(e^{-i \lambda t} u(t)\right) \text { as } T \rightarrow \infty .
\end{aligned}
$$

Hence it follows from (3.1) that

$$
\begin{aligned}
\lim _{T \rightarrow \infty} \frac{1}{T} \int_{0}^{T} e^{-i \lambda t} x^{\star} A u(t) d t & =\left(A^{\star} x^{\star}\right) m\left(e^{-i \lambda t} u(t)\right) \\
& =-\lambda^{2} x^{\star} m\left(e^{-i \lambda t} u(t)\right)-x^{\star} m\left(e^{-i \lambda t} f(t)\right) .
\end{aligned}
$$

Thus we have

$$
\text { - } x^{\star} m\left(e^{-i \lambda t} f(t)\right)=\left(\lambda^{2} x^{\star}+A^{\star} x^{\star}\right) m\left(e^{-i \lambda t} u(t)\right) \text {. }
$$

Now the rest of the proof parallels that of Theorem 1 . 
REMARK 2. The conclusion of Theorem 2 also remains valid if $D\left(A^{\star}\right)$ is total and $R\left(\lambda^{2}+A^{\star}\right)$ is total for all real $\lambda=0$, instead of dense in $X^{\star}$.

RENARK 3. If $X$ is a Hilbert space and $A$ is a nonnegative self-adjoint operator, then the hypotheses on A in Theorem 2 are verified (see Corollary 2, p. 208, Yosida [2]) and so Theorem 2 is a generalization of a result of Zaidman [3].

NOTE. As a consequence of our Theorem 1, we have the following result:

THEOREM 3. In a Hilbert space $H$, suppose $A$ is a self-adjoint operator and $f: J \rightarrow H$ is a weakly almost periodic (or an $S^{1}$-almost periodic continuous) function. If a differentiable function $u: J \rightarrow D(A)$ is a weakly almost periodic solution of the differential equation

$u^{\prime}(t)=A u(t)+f(t)$

on $J$, with $u^{\prime}$ being weakly continuous on $J$, then $\sigma(u(t)) \backslash\{0\}=\sigma(f(t)) \backslash\{0\}$.

PROOF. By Example 4, P. 210, Yosida [2], $R(i \lambda-A)=H$ for all real $\lambda \neq 0$.

ACKNOWLEDGEMENT. This work was supported by the National Research Council of Canada Grant Nos. 4056 and A-9085.

\section{REFERENCES}

1. AMERIO, L. and PROUSE, G. Almost Periodic Functions and Functional Equations, Van Nostrand Reinhold Company (1971).

2. YOSIDA, K. Functional Analysis, Springer-Verlag New York Inc. (1971).

3. ZAIDMAN, S. Spectrum of Almost Periodic Solutions for some abstract Differential Equations, Math. Anal. Appl., 28 (1969), pp. 336-338 


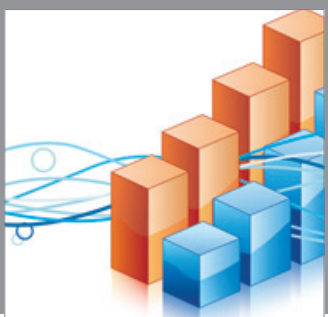

Advances in

Operations Research

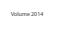

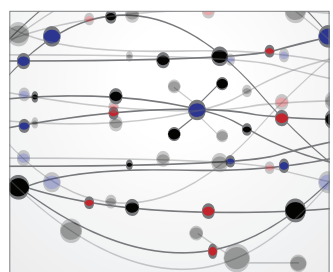

\section{The Scientific} World Journal
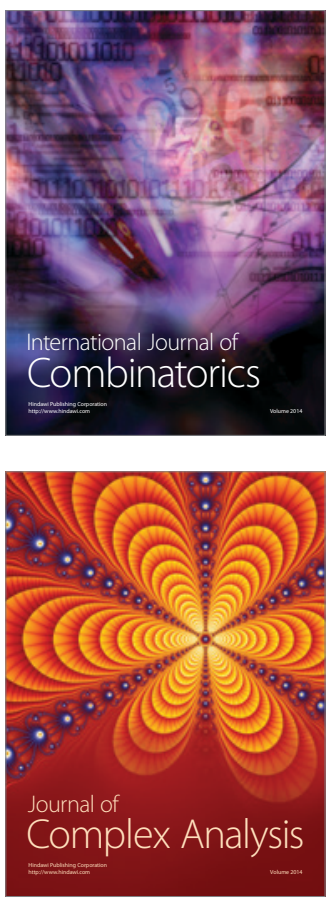

International Journal of

Mathematics and

Mathematical

Sciences
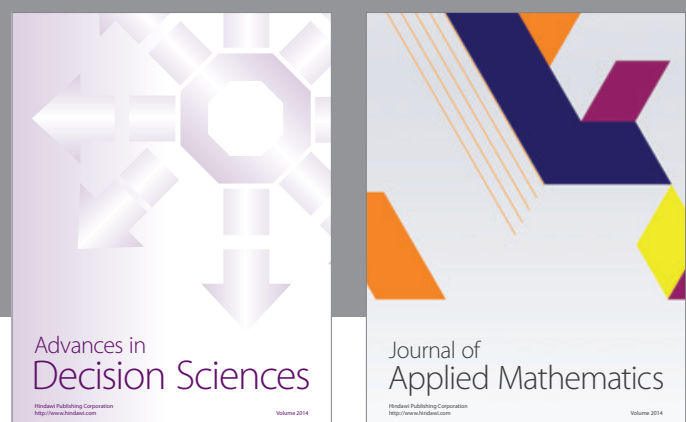

Journal of

Applied Mathematics
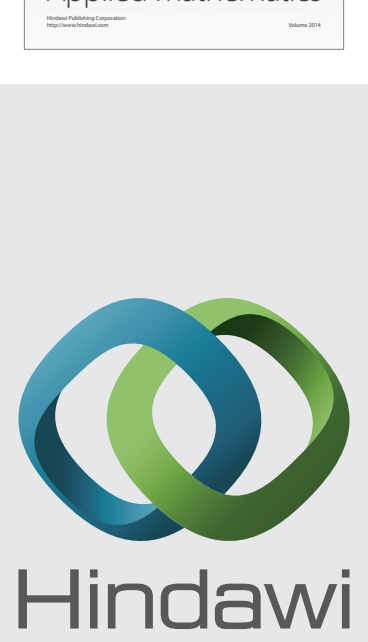

Submit your manuscripts at http://www.hindawi.com
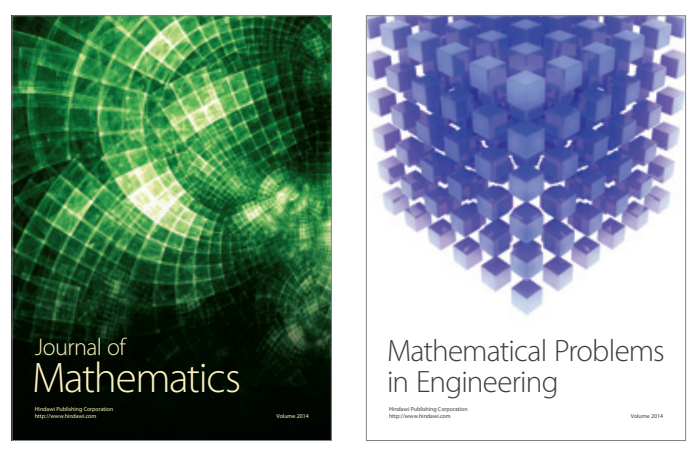

Mathematical Problems in Engineering
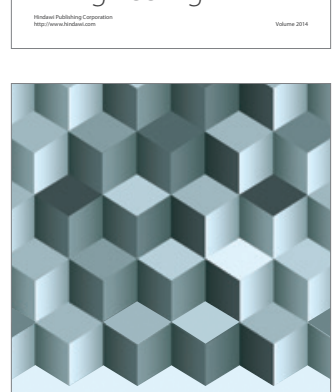

Journal of

Function Spaces
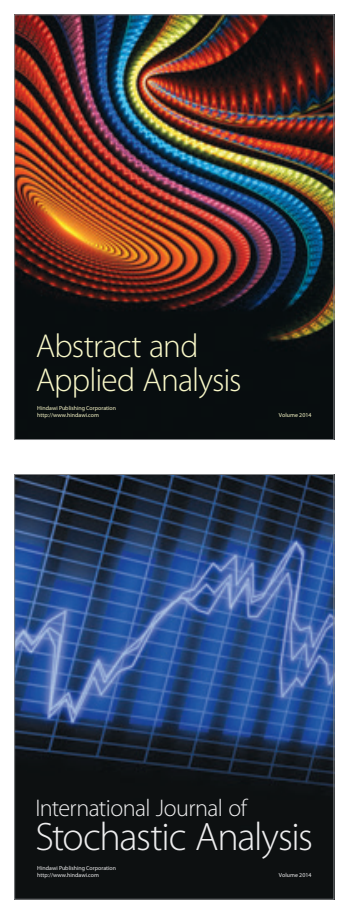

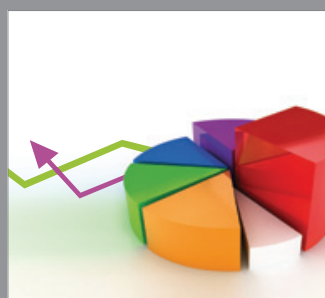

ournal of

Probability and Statistics

Promensencen
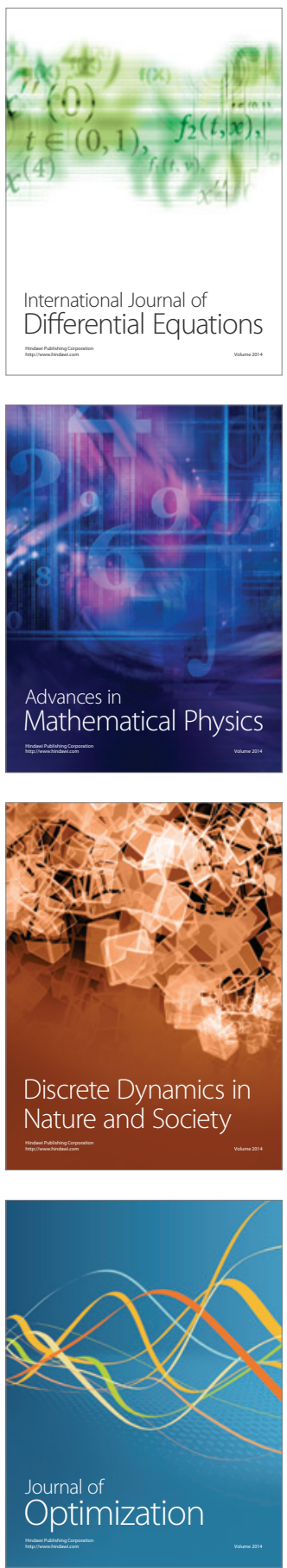